\title{
Common Fixed Point for Generalized Bose-Mukherjee-Type Fuzzy Mappings
}

\author{
Ming-liang Song and Zhong-qian Wang \\ Mathematics and Information Technology School, Jiangsu Second Normal University, Nanjing 210013, China \\ Correspondence should be addressed to Ming-liang Song; mlsong2004@163.com
}

Received 17 May 2013; Accepted 14 July 2013

Academic Editors: M. Rojas-Medar and X. Xue

Copyright ( 2013 M.-l. Song and Z.-q. Wang. This is an open access article distributed under the Creative Commons Attribution License, which permits unrestricted use, distribution, and reproduction in any medium, provided the original work is properly cited.

We prove a common fixed point theorem for a pair of generalized Bose-Mukherjee-type fuzzy mappings in a complete metric space. An example is also provided to support the main result presented herein.

\section{Introduction and Preliminaries}

In many scientific and engineering applications, the fuzzy set concept plays an important role. The concept of fuzzy sets was introduced initially by Zadeh [1] in 1965. Since then, the study of fixed point theorems in fuzzy mathematics had been instigated by Weiss [2] and Butnariu [3]. Heilpern [4] introduced the concept of fuzzy contractive mappings and proved a fixed point theorem for these mappings in metric linear spaces. His result is a generalization of the fixed point theorem for point-to-set maps of Nadler [5]. Afterwards, several fixed point theorems for fuzzy contractive mappings have appeared in the literature (see [6-13]). Particularly, Vijayaraju and Marudai [6] studied a fixed point result for fuzzy (multivalued) mappings $T: X \rightarrow \mathscr{F}(X)$ in a metric space $X$. This result [6] is significant as it does not require the condition of approximate quantity for $T(x)$ and linearity for X. However, Azam and Arshad [7] pointed out that its proof [6] is incorrect and incomplete and presented the right version of this result. In fact, although there exist mistakes in the proof of Theorem 3.1 in [6], its conclusion is correct.

The aim of this work is to establish a common fixed point theorem for a pair of generalized Bose-Mukherjee-type fuzzy mappings in a complete metric space. Also, we give an example to show the validity of our result and by which indicate that our result improves and extends several known results in $[6,7,14]$.

Let $X$ and $Y$ be nonempty sets. A multivalued mapping $T$ from $X$ to $Y$, denoted by $T: X \rightarrow 2^{Y}$, is defined to be a function that assigns to each element of $X$ a nonempty subset of $Y$. Fixed points of the multivalued mapping $T$ : $X \rightarrow 2^{X}$ will be the points $x \in X$ such that $x \in T(x)$.

Let $(X, d)$ be a metric space and let $\mathscr{C} \mathscr{B}(X)$ denote the set of all nonempty closed and bounded subsets of $X$. For $A, B \in$ $\mathscr{C} \mathscr{B}(X)$, define

$$
H(A, B)=\max \left\{\sup _{x \in A} d(x, B), \sup _{y \in B} d(A, y)\right\},
$$

where $d(x, A)=\inf _{y \in A} d(x, y)$.

A fuzzy set in $X$ is a function with domain $X$ and values in $[0,1]$. If $A$ is a fuzzy set and $x \in X$, then the function value $A(x)$ is called the grade of membership of $x$ in $A$. The $\alpha$-level set of $A$ is denoted by $[A]_{\alpha}$ and is defined as follows:

$$
\begin{gathered}
{[A]_{\alpha}=\{x: A(x) \geq \alpha\} \quad \text { if } \alpha \in(0,1],} \\
{[A]_{0}=\overline{\{x: A(x)>0\} .}}
\end{gathered}
$$

Here, $\bar{B}$ denotes the closure of the set $B$. Let $\mathscr{F}(X)$ be the collection of all fuzzy sets in a metric space $X$. For $A, B \in$ $\mathscr{F}(X), A \subset B$ means $A(x) \leq B(x)$ for each $x \in X$.

A mapping $T$ from $X$ to $\mathscr{F}(Y)$ is called a fuzzy mapping if for each $x \in X, T(x)$ (sometimes denoted by $T x$ ) is a fuzzy set on $Y$ and $T x(y)$ denotes the degree of membership of $y$ in $T x$. Let $\mathscr{W}(X)$ denote the set of all fuzzy sets on $X$ such that each of its $\alpha$-level is a nonempty closed bounded subset of $X$. 
The following lemmas are needed in the sequel.

Lemma 1 (Nadler [5]). Let $(X, d)$ be a metric space and $A, B \in$ $\mathscr{C} \mathscr{B}(X)$; then

(1) for each $x \in A, d(x, B) \leq H(A, B)$,

(2) for each $y \in X, d(x, A) \leq d(x, y)+d(y, A)$.

Lemma 2 (Nadler [5]). Let $(X, d)$ be a metric space and $A, B \in$ $\mathscr{C} \mathscr{B}(X)$; then for each $x \in A$ and $\varepsilon>0$ there exists an element $y \in B$ such that $d(x, y) \leq H(A, B)+\varepsilon$.

\section{Main Results}

Lemma 3. Let $A_{1}, A_{2}, A_{3}, A_{4}$, and $A_{5}$ be five nonnegative real numbers with $A_{1}+A_{2}+A_{3}+A_{4}+A_{5}=1, A_{5}>0$, and either $A_{1}>A_{2}, A_{3}>A_{4}$ or $A_{1}<A_{2}, A_{3}<A_{4}$. Let $a=\left(A_{1}+A_{3}+A_{5}\right) /\left(A_{1}+A_{4}+A_{5}\right), b=\left(A_{2}+A_{4}+A_{5}\right) /\left(A_{2}+\right.$ $\left.A_{3}+A_{5}\right)$; then $0<a b<1$.

Proof. If $A_{1}>A_{2}, A_{3}>A_{4}$, then $A_{1}>0, A_{3}>0$. Note that $A_{5}>0$; we have $a=\left(A_{1}+A_{3}+A_{5}\right) /\left(A_{1}+A_{4}+A_{5}\right)>0, b=$ $\left(A_{2}+A_{4}+A_{5}\right) /\left(A_{2}+A_{3}+A_{5}\right)>0$; that is, $a b>0$. Moreover, it is evident that $\left(A_{1}-A_{2}\right)\left(A_{3}-A_{4}\right)>0 \Rightarrow A_{1} A_{3}+A_{2} A_{4}>$ $A_{1} A_{4}+A_{2} A_{3}$, which further implies that

$$
\begin{aligned}
\left(A_{1}\right. & \left.+A_{4}+A_{5}\right)\left(A_{2}+A_{3}+A_{5}\right) \\
= & A_{1} A_{2}+A_{1} A_{3}+A_{1} A_{5}+A_{4} A_{2}+A_{4} A_{3} \\
& +A_{4} A_{5}+A_{5} A_{2}+A_{5} A_{3}+A_{5} A_{5} \\
> & A_{1} A_{2}+A_{1} A_{4}+A_{1} A_{5}+A_{3} A_{2}+A_{3} A_{4} \\
& +A_{3} A_{5}+A_{5} A_{2}+A_{5} A_{4}+A_{5} A_{5} \\
= & \left(A_{1}+A_{3}+A_{5}\right)\left(A_{2}+A_{4}+A_{5}\right) .
\end{aligned}
$$

That is, $a b=\left(A_{1}+A_{3}+A_{5}\right)\left(A_{2}+A_{4}+A_{5}\right) /\left(A_{1}+A_{4}+\right.$ $\left.A_{5}\right)\left(A_{2}+A_{3}+A_{5}\right)<1$.

Similarly, if $A_{1}<A_{2}, A_{3}<A_{4}$, then $0<a b<1$ holds.

Theorem 4. Let $(X, d)$ be a complete metric space. Let $S, T$ : $X \rightarrow \mathscr{F}(X)$ be two generalized Bose-Mukherjee-type fuzzy mappings. Suppose that, for each $x \in X$, there exists $\alpha(x) \in$ $(0,1]$ such that $[S x]_{\alpha(x)}$ and $[T x]_{\alpha(x)}$ are nonempty closed bounded subsets of $X$ and

$$
\begin{aligned}
H\left([S x]_{\alpha(x)},[T y]_{\alpha(y)}\right) \\
\quad \leq A_{1} d\left(x,[S x]_{\alpha(x)}\right)+A_{2} d\left(y,[T y]_{\alpha(y)}\right) \\
\quad+A_{3} d\left(x,[T y]_{\alpha(y)}\right)+A_{4} d\left(y,[S x]_{\alpha(x)}\right) \\
\quad+A_{5} d(x, y)
\end{aligned}
$$

for all $x, y \in X$, where $A_{1}, A_{2}, A_{3}, A_{4}$, and $A_{5}$ are five nonnegative real numbers with $\sum_{i=1}^{5} A_{i}=1, A_{5}>0$ and either $A_{1}>A_{2}, A_{3}>A_{4}$ or $A_{1}<A_{2}, A_{3}<A_{4}$. Then there exists $z \in X$ such that $z \in[S z]_{\alpha(z)} \cap[T z]_{\alpha(z)}$.
Proof. Let $A_{1}>A_{2}, A_{3}>A_{4}$ and $a=\left(A_{1}+A_{3}+A_{5}\right) /\left(A_{1}+\right.$ $\left.A_{4}+A_{5}\right), b=\left(A_{2}+A_{4}+A_{5}\right) /\left(A_{2}+A_{3}+A_{5}\right)$. By Lemma 3 , we know that $a>0, b>0$ and $0<a b<1$. Choosing $x_{0} \in X$, by hypotheses, there exists $\alpha\left(x_{0}\right) \in(0,1]$ such that $\left[S x_{0}\right]_{\alpha\left(x_{0}\right)}$ is nonempty closed bounded subset of $X$. For convenience, we denote $\alpha\left(x_{0}\right)$ by $\alpha_{1}$. Let $x_{1} \in\left[S x_{0}\right]_{\alpha_{1}}$; for this $x_{1}$ there exists $\alpha_{2} \in(0,1]$ such that $\left[T x_{1}\right]_{\alpha_{2}}$ is nonempty closed bounded subset of $X$. Since $A_{1}+A_{4}+A_{5}>0$, by Lemma 2 , there exists $x_{2} \in\left[T x_{1}\right]_{\alpha_{2}}$ such that

$$
d\left(x_{1}, x_{2}\right) \leq H\left(\left[S x_{0}\right]_{\alpha_{1}},\left[T x_{1}\right]_{\alpha_{2}}\right)+\left(A_{1}+A_{4}+A_{5}\right) .
$$

Since $A_{2}+A_{3}+A_{5}>0$, by the same argument, we can find $\alpha_{3} \in(0,1]$ and $x_{3} \in\left[S x_{2}\right]_{\alpha_{3}}$ such that

$$
d\left(x_{2}, x_{3}\right) \leq H\left(\left[S x_{2}\right]_{\alpha_{3}},\left[T x_{1}\right]_{\alpha_{2}}\right)+a b\left(A_{2}+A_{3}+A_{5}\right) .
$$

By induction, we produce a sequence $\left\{x_{n}\right\}$ of points of $X$,

$$
x_{2 k+1} \in\left[S x_{2 k}\right]_{\alpha_{2 k+1}}, \quad x_{2 k+2} \in\left[T x_{2 k+1}\right]_{\alpha_{2 k+2}}, \quad k=0,1,2, \ldots,
$$

such that

$$
\begin{aligned}
d\left(x_{2 k+1}, x_{2 k+2}\right) \leq & H\left(\left[S x_{2 k}\right]_{\alpha_{2 k+1}},\left[T x_{2 k+1}\right]_{\alpha_{2 k+2}}\right) \\
& +(a b)^{k}\left(A_{1}+A_{4}+A_{5}\right), \\
d\left(x_{2 k+2}, x_{2 k+3}\right) \leq & H\left(\left[S x_{2 k+2}\right]_{\alpha_{2 k+3}},\left[T x_{2 k+1}\right]_{\alpha_{2 k+2}}\right) \\
& +(a b)^{k+1}\left(A_{2}+A_{3}+A_{5}\right) .
\end{aligned}
$$

For $k=0,1,2, \ldots$, applying (4), we obtain

$$
\begin{aligned}
& d\left(x_{2 k+1}, x_{2 k+2}\right) \\
& \leq H\left(\left[S x_{2 k}\right]_{\alpha_{2 k+1}},\left[T x_{2 k+1}\right]_{\alpha_{2 k+2}}\right)+(a b)^{k}\left(A_{1}+A_{4}+A_{5}\right) \\
& \leq A_{1} d\left(x_{2 k},\left[S x_{2 k}\right]_{\alpha_{2 k+1}}\right)+A_{2} d\left(x_{2 k+1},\left[T x_{2 k+1}\right]_{\alpha_{2 k+2}}\right) \\
&+A_{3} d\left(x_{2 k},\left[T x_{2 k+1}\right]_{\alpha_{2 k+2}}\right)+A_{4} d\left(x_{2 k+1},\left[S x_{2 k}\right]_{\alpha_{2 k+1}}\right) \\
&+A_{5} d\left(x_{2 k}, x_{2 k+1}\right)+(a b)^{k}\left(A_{1}+A_{4}+A_{5}\right) \\
& \leq A_{1} d\left(x_{2 k}, x_{2 k+1}\right)+A_{2} d\left(x_{2 k+1}, x_{2 k+2}\right)+A_{3} d\left(x_{2 k}, x_{2 k+2}\right) \\
&+A_{4} d\left(x_{2 k+1}, x_{2 k+1}\right)+A_{5} d\left(x_{2 k}, x_{2 k+1}\right) \\
&+(a b)^{k}\left(A_{1}+A_{4}+A_{5}\right) \\
& \leq A_{1} d\left(x_{2 k}, x_{2 k+1}\right)+A_{2} d\left(x_{2 k+1}, x_{2 k+2}\right) \\
&+A_{3} d\left(x_{2 k}, x_{2 k+1}\right)+A_{3} d\left(x_{2 k+1}, x_{2 k+2}\right) \\
&+A_{5} d\left(x_{2 k}, x_{2 k+1}\right)+(a b)^{k}\left(A_{1}+A_{4}+A_{5}\right) .
\end{aligned}
$$


It implies that

$$
\begin{aligned}
d\left(x_{2 k+1}, x_{2 k+2}\right) \leq & \frac{A_{1}+A_{3}+A_{5}}{1-A_{2}-A_{3}} d\left(x_{2 k}, x_{2 k+1}\right) \\
& +(a b)^{k} \frac{A_{1}+A_{4}+A_{5}}{1-A_{2}-A_{3}} .
\end{aligned}
$$

Note that $\sum_{i=1}^{5} A_{i}=1$; we have

$$
\begin{aligned}
d\left(x_{2 k+1}, x_{2 k+2}\right) \leq & \frac{A_{1}+A_{3}+A_{5}}{A_{1}+A_{4}+A_{5}} d\left(x_{2 k}, x_{2 k+1}\right) \\
& +(a b)^{k} \frac{A_{1}+A_{4}+A_{5}}{A_{1}+A_{4}+A_{5}} \\
= & a d\left(x_{2 k}, x_{2 k+1}\right)+(a b)^{k} .
\end{aligned}
$$

Similarly, we have

$$
d\left(x_{2 k+2}, x_{2 k+3}\right) \leq b d\left(x_{2 k+1}, x_{2 k+2}\right)+(a b)^{k+1} .
$$

Using inductive method, for $k=0,1,2, \ldots$, by (11) and (12), we can obtain

$$
\begin{aligned}
d( & \left.x_{2 k+1}, x_{2 k+2}\right) \\
& \leq a d\left(x_{2 k}, x_{2 k+1}\right)+(a b)^{k} \\
& \leq a b d\left(x_{2 k-1}, x_{2 k}\right)+a(a b)^{k}+(a b)^{k} \\
& \leq a b a d\left(x_{2 k-2}, x_{2 k-1}\right)+(a b)^{k}+a(a b)^{k}+(a b)^{k} \\
& \leq(a b)^{2} d\left(x_{2 k-3}, x_{2 k-2}\right)+2(a+1)(a b)^{k} \\
& \leq \cdots \leq(a b)^{k} d\left(x_{1}, x_{2}\right)+k(a+1)(a b)^{k} \\
& \leq(a b)^{k} a d\left(x_{0}, x_{1}\right)+(a b)^{k}+k(a+1)(a b)^{k} \\
& \leq(a b)^{k} d\left(x_{0}, x_{1}\right)+(k+1)(a+1)(a b)^{k}, \\
d( & \left.x_{2 k+2}, x_{2 k+3}\right) \\
& \leq b\left((a b)^{k} a d\left(x_{0}, x_{1}\right)+(k+1)(a+1)(a b)^{k}\right)+(a b)^{k+1} \\
& =(a b)^{k+1} d\left(x_{0}, x_{1}\right)+b(k+1)(a+1)(a b)^{k}+(a b)^{k+1} \\
& \leq(a b)^{k+1} d\left(x_{0}, x_{1}\right)+(k+1)\left(\frac{a+1}{a}\right)(a b)^{k+1}+(a b)^{k+1} \\
& \\
& \left(a+1 d\left(x_{0}, x_{1}\right)+(k+2)\left(\frac{a+1}{a}\right)(a b)^{k+1} \cdot\right.
\end{aligned}
$$

Next, we prove that the sequence $\left\{x_{n}\right\}$ is a Cauchy sequence in $X$. For $k<p$, we have

$$
\begin{aligned}
& d\left(x_{2 k+1}, x_{2 p+1}\right) \\
& \leq d\left(x_{2 k+1}, x_{2 k+2}\right)+\cdots+d\left(x_{2 p}, x_{2 p+1}\right) \\
& \quad \leq\left(\sum_{i=k}^{p-1}(a b)^{i}+\sum_{i=k+1}^{p}(a b)^{i}\right) d\left(x_{0}, x_{1}\right)
\end{aligned}
$$

$$
\begin{aligned}
& +\left((a+1) \sum_{i=k}^{p-1}(i+1)(a b)^{i}+\left(\frac{a+1}{a}\right) \sum_{i=k+1}^{p}(i+1)(a b)^{i}\right) \\
\leq & \left(\frac{(a b)^{k}}{1-a b}+\frac{(a b)^{k+1}}{1-a b}\right) d\left(x_{0}, x_{1}\right) \\
& +\left((a+1) \sum_{i=k}^{\infty}(i+1)(a b)^{i}+\left(\frac{a+1}{a}\right) \sum_{i=k+1}^{\infty}(i+1)(a b)^{i}\right) \\
\leq & 2 \frac{(a b)^{k}}{1-a b} d\left(x_{0}, x_{1}\right)+2 M \sum_{i=k}^{\infty}(i+1)(a b)^{i},
\end{aligned}
$$

where $M=\max \{a+1,(a+1) / a\}$. By the similar reasoning process, we can obtain

$$
\begin{aligned}
& d\left(x_{2 k}, x_{2 p+1}\right) \leq 2 \frac{(a b)^{k}}{1-a b} d\left(x_{0}, x_{1}\right)+2 M \sum_{i=k}^{\infty}(i+1)(a b)^{i}, \\
& d\left(x_{2 k}, x_{2 p}\right) \leq 2 \frac{(a b)^{k}}{1-a b} d\left(x_{0}, x_{1}\right)+2 M \sum_{i=k}^{\infty}(i+1)(a b)^{i}, \\
& d\left(x_{2 k+1}, x_{2 p}\right) \leq 2 \frac{(a b)^{k}}{1-a b} d\left(x_{0}, x_{1}\right)+2 M \sum_{i=k}^{\infty}(i+1)(a b)^{i} .
\end{aligned}
$$

Then, there exists $k$ with $(n-1) / 2 \leq k \leq n / 2$, for any $0<n<$ $m$, such that

$$
d\left(x_{m}, x_{n}\right) \leq 2 \frac{(a b)^{k}}{1-a b} d\left(x_{0}, x_{1}\right)+2 M \sum_{i=k}^{\infty}(i+1)(a b)^{i} \text {. }
$$

Since $0<a b<1$, it follows from Cauchy's root test that $\Sigma(n+$ 1) $(a b)^{n}$ is convergent and hence $\left\{x_{n}\right\}$ is a Cauchy sequence in $X$. Since $X$ is a complete metric space, then there exists $z \in X$ such that $x_{n} \rightarrow z$ as $n \rightarrow \infty$. Then, by (4) and Lemma 1, we have

$$
\begin{aligned}
& d\left(z,[T(z)]_{\alpha(z)}\right) \\
& \leq d\left(z, x_{2 n+1}\right)+d\left(x_{2 n+1},[T(z)]_{\alpha(z)}\right) \\
& \leq d\left(z, x_{2 n+1}\right)+H\left(\left[S x_{2 n}\right]_{\alpha_{2 n+1}},[T(z)]_{\alpha(z)}\right) \\
& \leq d\left(z, x_{2 n+1}\right)+A_{1} d\left(x_{2 n},\left[S x_{2 n}\right]_{\alpha_{2 n+1}}\right) \\
&+A_{2} d\left(z,[T(z)]_{\alpha(z)}\right)+A_{3} d\left(x_{2 n},[T(z)]_{\alpha(z)}\right) \\
&+A_{4} d\left(z,\left[S x_{2 n}\right]_{\alpha_{2 n+1}}\right)+A_{5} d\left(x_{2 n}, z\right) \\
& \leq d\left(z, x_{2 n+1}\right)+A_{1} d\left(x_{2 n}, x_{2 n+1}\right)+A_{2} d\left(z,[T(z)]_{\alpha(z)}\right) \\
&+A_{3} d\left(x_{2 n}, z\right)+A_{3} d\left(z,[T(z)]_{\alpha(z)}\right)+A_{4} d\left(z, x_{2 n+1}\right) \\
&+A_{5} d\left(x_{2 n}, z\right) .
\end{aligned}
$$


Therefore,

$$
\begin{aligned}
(1- & \left.A_{2}-A_{3}\right) d\left(z,[T(z)]_{\alpha(z)}\right) \\
\leq & d\left(z, x_{2 n+1}\right)+A_{1} d\left(x_{2 n}, x_{2 n+1}\right) \\
& +A_{3} d\left(x_{2 n}, z\right)+A_{4} d\left(z, x_{2 n+1}\right) \\
& +A_{5} d\left(x_{2 n}, z\right)
\end{aligned}
$$

and hence $d\left(z,[T(z)]_{\alpha(z)}\right) \rightarrow 0$ as $n \rightarrow \infty$. Thus, $z \in$ $[T z]_{\alpha(z)}$.

Similarly, we can prove that $z \in[S z]_{\alpha(z)}$. Hence, $z \in$ $[S z]_{\alpha(z)} \cap[T z]_{\alpha(z)}$.

If $A_{1}<A_{2}, A_{3}<A_{4}$, by the same argument, we can prove that the conclusion holds.

Corollary 5 (Vijayaraju and Marudai [6]). Let $(X, d)$ be a complete metric space. Let $S, T: X \rightarrow \mathscr{F}(X)$ be two fuzzy mappings. Suppose that, for each $x \in X$, there exists $\alpha(x) \in(0,1]$ such that $[S x]_{\alpha(x)}$ and $[T x]_{\alpha(x)}$ are nonempty closed bounded subsets of $X$ and

$$
\begin{aligned}
& H\left([S x]_{\alpha(x)},[T y]_{\alpha(y)}\right) \\
& \quad \leq a_{1} d\left(x,[S x]_{\alpha(x)}\right)+a_{2} d\left(y,[T y]_{\alpha(y)}\right) \\
& \quad+a_{3} d\left(x,[T y]_{\alpha(y)}\right)+a_{4} d\left(y,[S x]_{\alpha(x)}\right)+a_{5} d(x, y)
\end{aligned}
$$

for all $x, y \in X$, where $a_{1}, a_{2}, a_{3}, a_{4}$, and $a_{5}$ are nonnegative real numbers with $\sum_{i=1}^{5} a_{i}<1$ and either $a_{1}=a_{2}$ or $a_{3}=a_{4}$. Then, there exists $z \in X$ such that $z \in[S z]_{\alpha(z)} \cap[T z]_{\alpha(z)}$.

Proof. If $a_{1}=a_{2}, a_{3}>a_{4}$, by $\sum_{i=1}^{5} a_{i}<1$, we can take $2 \delta=1-$ $\left(\sum_{i=1}^{5} a_{i}\right)>0$. And let $A_{1}=a_{1}+\delta, A_{2}=a_{2}, A_{3}=a_{3}, A_{4}=a_{4}$, and $A_{5}=a_{5}+\delta$; then we have $\Sigma_{i=1}^{5} A_{i}=1, A_{1}>A_{2}, A_{3}>$ $A_{4}, A_{5}>0$, and for all $x, y \in X$,

$$
\begin{aligned}
H( & {\left.[S x]_{\alpha(x)},[T y]_{\alpha(y)}\right) } \\
\quad \leq & A_{1} d\left(x,[S x]_{\alpha(x)}\right)+A_{2} d\left(y,[T y]_{\alpha(y)}\right) \\
& +A_{3} d\left(x,[T y]_{\alpha(y)}\right)+A_{4} d\left(y,[S x]_{\alpha(x)}\right)+A_{5} d(x, y),
\end{aligned}
$$

which implies the conditions of Theorem 4 are satisfied. Similarly, we can prove that some cases of $a_{1}=a_{2}, a_{3}<a_{4}$ or $a_{1}>a_{2}, a_{3}=a_{4}$ or $a_{1}<a_{2}, a_{3}=a_{4}$, respectively. Therefore, by Theorem 4 , the corollary is proved.

Remark 6. Corollary 5 shows that, although there exist mistakes in the proof of Theorem 3.1 in [6], its conclusion is correct. Moreover, Corollary 5 also shows that Theorem 4 in [7] is not the right version of Theorem 3.1 in [6] but the special case of Theorem 3.1 in [6]. In addition, we give a correct proof of Theorem 3.1 in [6].

Corollary 7 (Azam and Arshad [7]). Let $(X, d)$ be a complete metric space. Let $S, T: X \rightarrow \mathscr{F}(X)$ be two fuzzy mappings.
Suppose that, for each $x \in X$, there exists $\alpha(x) \in(0,1]$ such that $[S x]_{\alpha(x)}$ and $[T x]_{\alpha(x)}$ are nonempty closed bounded subsets of $X$ and

$$
\begin{aligned}
& H\left([S x]_{\alpha(x)},[T y]_{\alpha(y)}\right) \\
& \quad \leq a_{1} d\left(x,[S x]_{\alpha(x)}\right)+a_{2} d\left(y,[T y]_{\alpha(y)}\right) \\
& \quad+a_{3}\left[d\left(x,[T y]_{\alpha(y)}\right)+d\left(y,[S x]_{\alpha(x)}\right)\right]+a_{4} d(x, y)
\end{aligned}
$$

for all $x, y \in X$, where $a_{1}, a_{2}, a_{3}$, and $a_{4}$ are nonnegative real numbers with $a_{1}+a_{2}+2 a_{3}+a_{4}<1$. Then, there exists $z \in X$ such that $z \in[S z]_{\alpha(z)} \cap[T z]_{\alpha(z)}$.

Theorem 8. Let $(X, d)$ be a complete metric space. Let $S, T$ : $X \rightarrow \mathscr{C} \mathscr{B}(X)$ be two generalized Bose-Mukherjee-type multivalued mappings. Suppose that, for all $x, y \in X$,

$$
\begin{aligned}
H(S x, T y) \leq & A_{1} d(x, S x)+A_{2} d(y, T y) \\
& +A_{3} d(x, T y)+A_{4} d(y, S x) \\
& +A_{5} d(x, y),
\end{aligned}
$$

where $A_{1}, A_{2}, A_{3}, A_{4}$, and $A_{5}$ are five nonnegative real numbers with $\Sigma_{i=1}^{5} A_{i}=1, A_{5}>0$ and either $A_{1}>A_{2}, A_{3}>$ $A_{4}$ or $A_{1}<A_{2}, A_{3}<A_{4}$. Then, there exists $z \in X$ such that $z \in S z \cap T z$.

Proof. Let the fuzzy mappings $S, T: X \rightarrow \mathscr{F}(X)$ be defined as $S(x)=\chi_{S(x)}$ and $T(x)=\chi_{T(x)}$, where $\chi_{A}$ is the characteristic function on any subset $A$ of $X$. Using the facts $[S x]_{\alpha(x)}=S(x)$ and $[T x]_{\alpha(x)}=T(x)$ for any $\alpha(x) \epsilon$ $(0,1]$, it is evident that $S$ and $T$ satisfy the condition of Theorem 4 .

Similarly, as the proof of Corollary 5, from Theorem 8 , we can obtain that common fixed point theorem for BoseMukherjee-type multivalued mappings in [14].

Corollary 9 (Bose and Mukherjee [14]). Let $(X, d)$ be a complete metric space. Let $S, T: X \rightarrow \mathscr{C} \mathscr{B}(X)$ be two multivalued mappings. Suppose that, for all $x, y \in X$,

$$
\begin{aligned}
H(S x, T y) \leq & a_{1} d(x, S x)+a_{2} d(y, T y) \\
& +a_{3} d(x, T y)+a_{4} d(y, S x) \\
& +a_{5} d(x, y)
\end{aligned}
$$

where $a_{1}, a_{2}, a_{3}, a_{4}$, and $a_{5}$ are nonnegative real numbers with $\sum_{i=1}^{5} a_{i}<1$ and either $a_{1}=a_{2}$ or $a_{3}=a_{4}$. Then, there exists $z \in X$ such that $z \in S z \cap T z$. 
Example 10. Let $X=\{0,1,2\} ; d$ is an ordinary metric; then $(X, d)$ is a complete metric space. Define two fuzzy mappings $S, T: X \rightarrow \mathscr{F}(X)$ as follows:

$$
(S x)(z)=\left\{\begin{array}{ll}
1, & \text { if } z=0, \\
0, & \text { if } z=1 \text { or } 2,
\end{array} \quad \forall x \in X\right.
$$

$(T 2)(z)=\left\{\begin{array}{ll}1, & \text { if } z=1, \\ 0, & \text { if } z=0 \text { or } 2,\end{array}\right.$ and for $y \in X \backslash\{2\}$,

$$
(T y)(z)= \begin{cases}1, & \text { if } z=0 \\ 0, & \text { if } z=1 \text { or } 2\end{cases}
$$

Then, we have

$$
\begin{gathered}
{[S x]_{1}=[S x]_{\alpha}=\{0\} \quad \forall x \in X, \alpha \in(0,1],} \\
{[T y]_{1}=[T y]_{\alpha}=\left\{\begin{array}{ll}
\{1\}, & \text { if } y=2, \\
\{0\}, & \text { if } y=0 \text { or } 1,
\end{array} \quad \forall \alpha \in(0,1] .\right.}
\end{gathered}
$$

Now we take $A_{1}=2 / 45, A_{2}=22 / 45, A_{3}=1 / 9, A_{4}=23 /$ 90, $A_{5}=1 / 10$; then we have $\sum_{i=1}^{5} A_{i}=1, A_{5}>0$ and $A_{1}<$ $A_{2}, A_{3}<A_{4}$.

Moreover, if $x \in X$ and $y=0$ or 1 , then, for all $\alpha \in(0,1]$,

$$
\begin{aligned}
H\left([S x]_{\alpha},[T y]_{\alpha}\right)= & 0 \\
\leq & \frac{2}{45} d\left(x,[S x]_{\alpha}\right)+\frac{22}{45} d\left(y,[T y]_{\alpha}\right) \\
& +\frac{1}{9} d\left(x,[T y]_{\alpha}\right)+\frac{23}{90} d\left(y,[S x]_{\alpha}\right) \\
& +\frac{1}{10} d(x, y) .
\end{aligned}
$$

If $x=0$ and $y=2$, then, for all $\alpha \in(0,1]$,

$$
\begin{aligned}
H\left([S 0]_{\alpha},[T 2]_{\alpha}\right)= & 1 \\
< & \frac{2}{45} d(0,\{0\})+\frac{22}{45} d(2,\{1\}) \\
& +\frac{1}{9} d(0,\{1\})+\frac{23}{90} d(2,\{0\})+\frac{1}{10} d(0,2) .
\end{aligned}
$$

If $x=1$ and $y=2$, then, for all $\alpha \in(0,1]$,

$$
\begin{aligned}
H\left([S 1]_{\alpha},[T 2]_{\alpha}\right)= & 1 \\
< & \frac{2}{45} d(1,\{0\})+\frac{22}{45} d(2,\{1\}) \\
& +\frac{1}{9} d(1,\{1\})+\frac{23}{90} d(2,\{0\})+\frac{1}{10} d(1,2) .
\end{aligned}
$$

If $x=2$ and $y=2$, then, for all $\alpha \in(0,1]$,

$$
\begin{aligned}
H\left([S 2]_{\alpha},[T 2]_{\alpha}\right)= & 1 \\
< & \frac{2}{45} d(2,\{0\})+\frac{22}{45} d(2,\{1\}) \\
& +\frac{1}{9} d(2,\{1\})+\frac{23}{90} d(2,\{0\})+\frac{1}{10} d(2,2) .
\end{aligned}
$$

Hence, the conditions of Theorem 4 are satisfied, and there exists $0 \in X$ such that $0 \in\{0\}=[S 0]_{\alpha} \cap[T 0]_{\alpha}$. But, for any nonnegative real numbers $a_{1}, a_{2}, a_{3}, a_{4}$, and $a_{5}$ with $a_{1}+a_{2}+$ $2 a_{3}+a_{5}<1$, we have

$$
\begin{aligned}
H\left([S 1]_{\alpha},[T 2]_{\alpha}\right)= & 1 \\
> & a_{1} d(1,\{0\})+a_{2} d(2,\{1\}) \\
& +a_{3}[d(1,\{1\})+d(2,\{0\})]+a_{5} d(1,2)
\end{aligned}
$$

for all $\alpha \in(0,1]$. Thus, $S, T$ cannot satisfy the general contractive condition $a_{1}+a_{2}+2 a_{3}+a_{5}<1$.

\section{Conclusion}

The aim of this work is to establish a common fixed point theorem for a pair of generalized Bose-Mukherjee-type fuzzy mappings in a complete metric space. Also, we give an example to show the validity of our result and by which indicate that our result improves and extends several known results in $[6,7,14]$. Moreover, we give a correct proof of Theorem 3.1 in [6] and point out the conclusion of Theorem 4.1 in [7] is the special case of Theorem 3.1 in [6]. Finally, we hope that this theory would provide a mathematical background to the ongoing work in the problems of scientific and engineering applications.

\section{Acknowledgments}

This work was supported by the Natural Science Foundation of the Jiangsu Higher Education Institutions (Grant no. 13KJB110004) and Qing Lan Project of Jiangsu Province of China.

\section{References}

[1] L. A. Zadeh, "Fuzzy sets," Information and Computation, vol. 8, pp. 338-353, 1965.

[2] M. D. Weiss, "Fixed points, separation, and induced topologies for fuzzy sets," Journal of Mathematical Analysis and Applications, vol. 50, pp. 142-150, 1975.

[3] D. Butnariu, "Fixed points for fuzzy mappings," Fuzzy Sets and Systems, vol. 7, no. 2, pp. 191-207, 1982.

[4] S. Heilpern, "Fuzzy mappings and fixed point theorem," Journal of Mathematical Analysis and Applications, vol. 83, no. 2, pp. 566-569, 1981.

[5] S. B. Nadler Jr., "Multi-valued contraction mappings", Pacific Journal of Mathematics, vol. 30, pp. 475-488, 1969. 
[6] P. Vijayaraju and M. Marudai, "Fixed point theorems for fuzzy mappings," Fuzzy Sets and Systems, vol. 135, no. 3, pp. 401-408, 2003.

[7] A. Azam and M. Arshad, "A note on "Fixed point theorems for fuzzy mappings” by P. Vijayaraju and M. Marudai," Fuzzy Sets and Systems, vol. 161, no. 8, pp. 1145-1149, 2010.

[8] H. M. Abu-Donia, "Common fixed point theorems for fuzzy mappings in metric space under $\varphi$-contraction condition," Chaos, Solitons \& Fractals, vol. 34, no. 2, pp. 538-543, 2007.

[9] T. Kamran, "Common fixed points theorems for fuzzy mappings," Chaos, Solitons and Fractals, vol. 38, no. 5, pp. 1378-1382, 2008.

[10] A. Azam and I. Beg, "Common fixed points of fuzzy maps," Mathematical and Computer Modelling, vol. 49, no. 7-8, pp. 13311336, 2009.

[11] B. S. Lee, G. M. Lee, S. J. Cho, and D. S. Kim, "A common fixed point theorem for a pair of fuzzy mappings," Fuzzy Sets and Systems, vol. 98, no. 1, pp. 133-136, 1998.

[12] T. Som and R. N. Mukherjee, "Some fixed point theorems for fuzzy mappings," Fuzzy Sets and Systems, vol. 33, no. 2, pp. 213219, 1989.

[13] H. Román-Flores, A. Flores-Franulic, M. Rojas-Medar, and R. C. Bassanezi, "Stability of fixed points set of fuzzy contractions," Applied Mathematics Letters, vol. 11, no. 4, pp. 33-37, 1998.

[14] R. K. Bose and R. N. Mukherjee, "Common fixed points of some multi-valued mappings," Tamkang Journal of Mathematics, vol. 8, no. 2, pp. 245-249, 1977. 


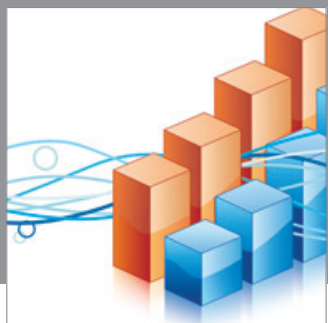

Advances in

Operations Research

mansans

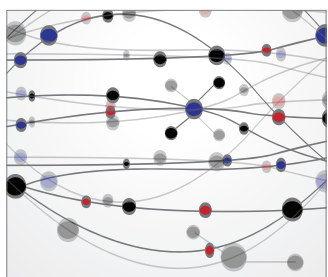

The Scientific World Journal
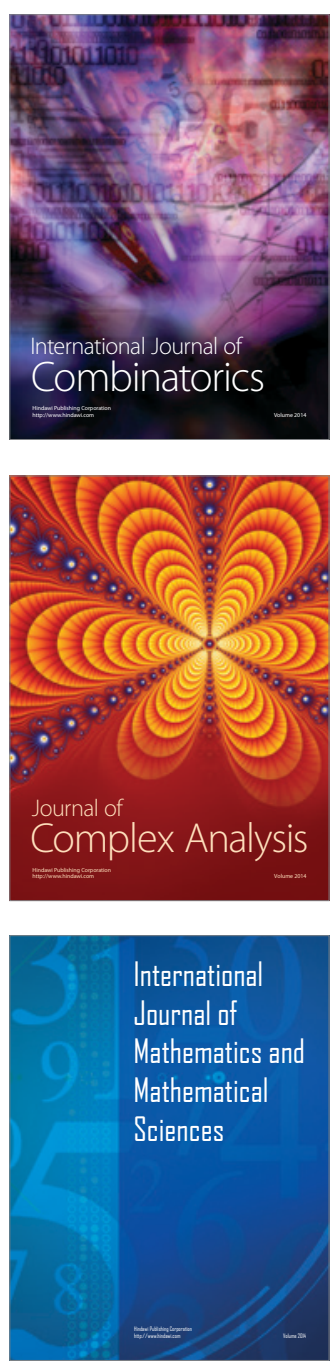
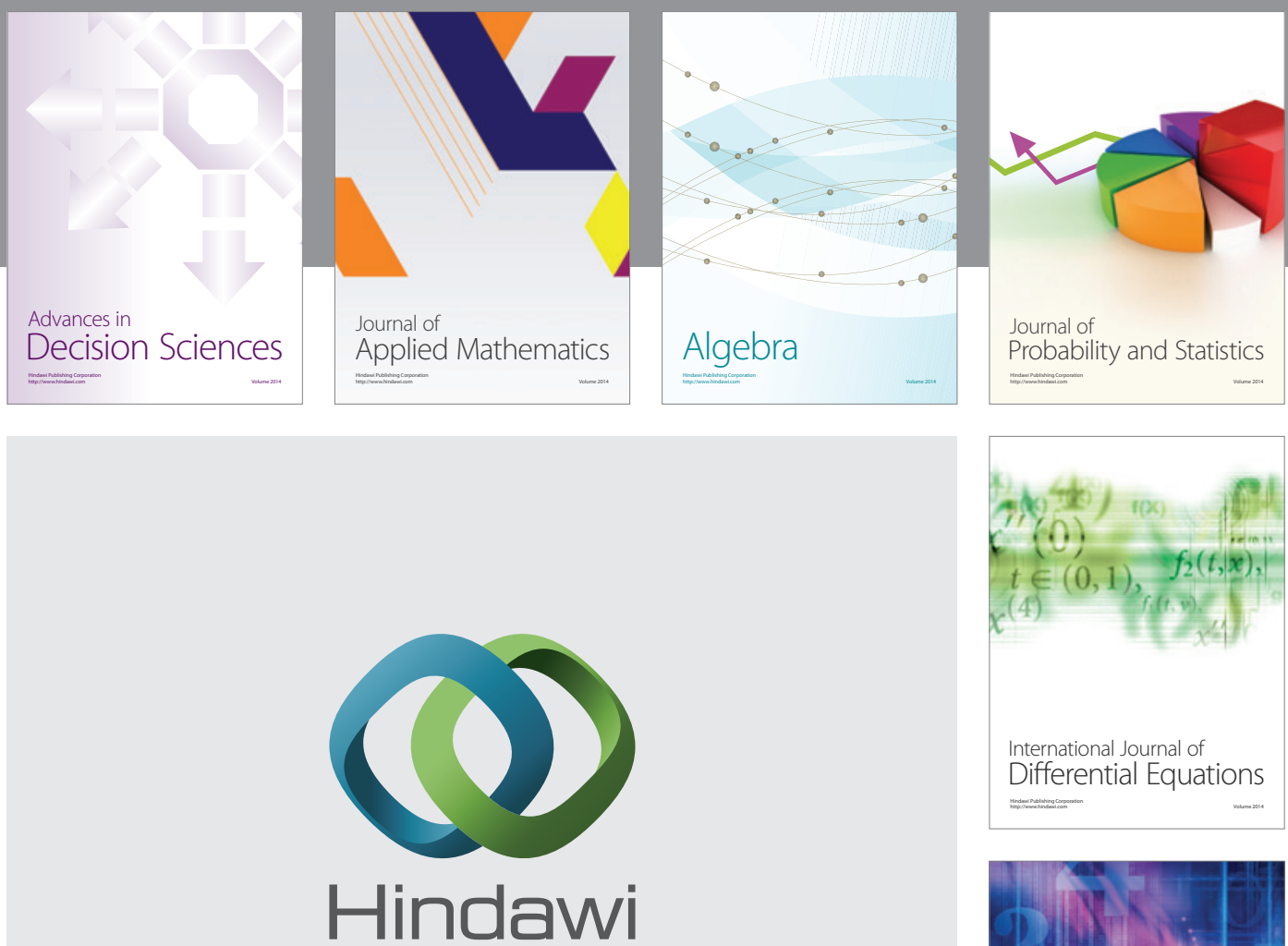

Submit your manuscripts at http://www.hindawi.com
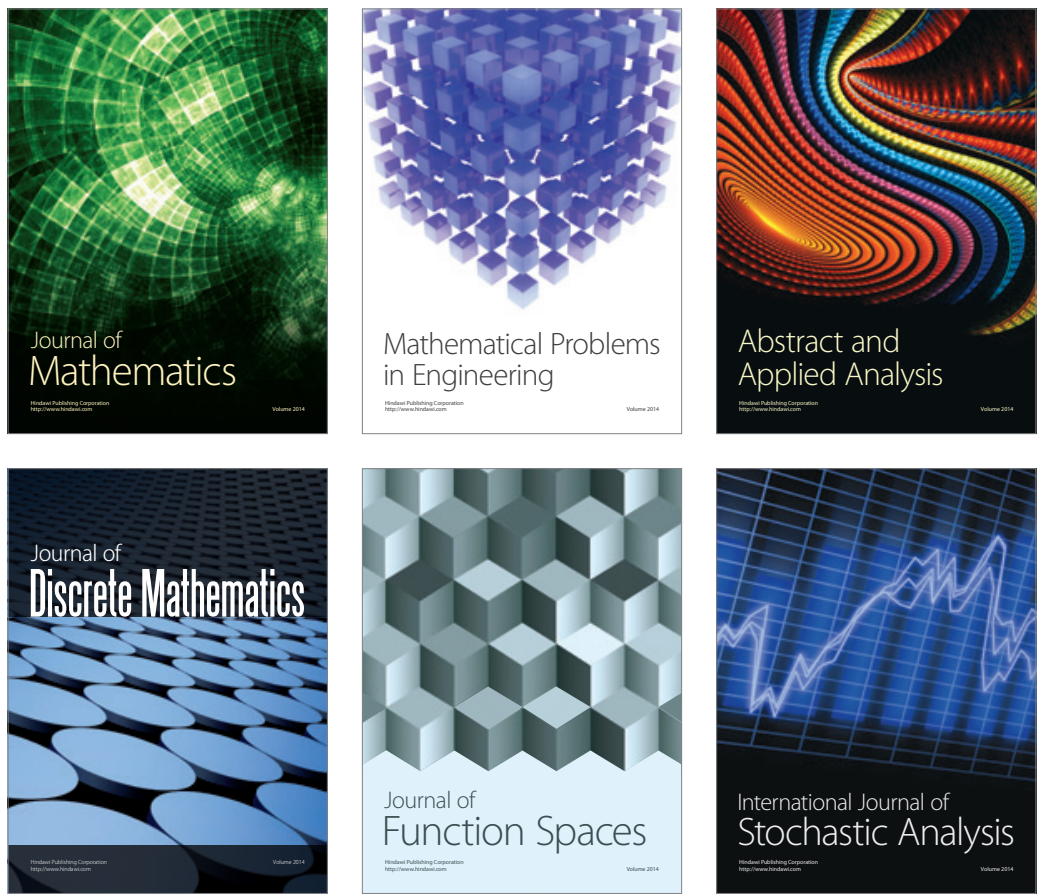

Journal of

Function Spaces

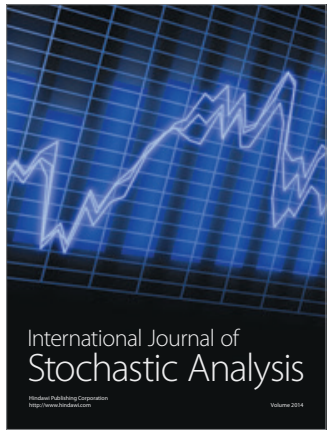

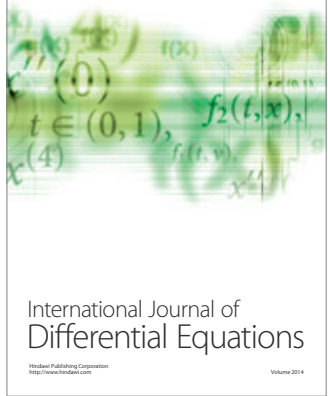
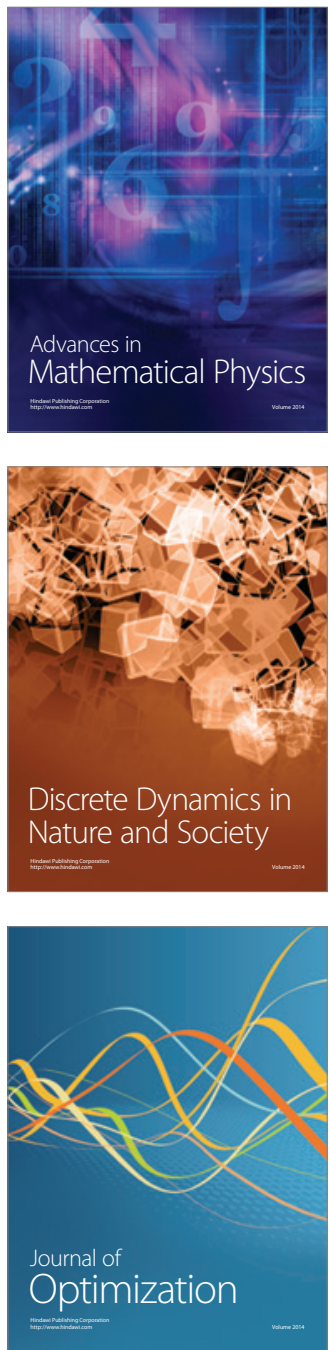